\section{Postmortem medicolegal genetic diagnostics also require reporting guidance}

European Journal of Human Genetics (2016) 24, 329-330; doi:10.1038/ejhg.2014.247; published online 3 December 2014

The recommendations by the working group (WG) of the Genetic Services Quality Committee of the European Society of Human Genetics (ESHG) for reporting results of diagnostic genetic testing (biochemical, cytogenetic and molecular genetic) published by Claustres et al in the European Journal of Human Genetics are welcomed and definitely needed. The WG focused on clinical patients, but did not address reporting of genetic testing in deceased individuals, especially for those who have been subjected to a medicolegal autopsy. In the realm of medicolegal autopsy practice, samples are collected routinely for human identification purposes. However, beyond identity testing, there are cases where genetic diagnostics are being performed to establish the underlying or contributing causes (and manners) of death. Although conceptually still somewhat in its infancy, 'molecular autopsy' employs genetic markers and predictive power of risk to assist in cause and manner of death investigations. ${ }^{2}$ The situations often considered include genetic testing in negative autopsies, that is, sudden unexpected deaths, that have no findings in standard autopsies and cases with unusual toxicology results (pharmacogenetic analyses). ${ }^{3,4}$ At the same time, national legislations for reporting of genetic tests postmortem are diverse or nonexistent and, although some local practice guidelines have been established, ${ }^{5}$ international recommendations concerning medicolegal and forensic postmortem genetic diagnostics are lacking.

The principles and recommendations advocated by the ESHG WG also provide a good framework by which to guide the reporting of postmortem genetic tests. However, the medicolegal setting encounters some aspects that differ from the standard clinical arena. First, a medicolegal autopsy, in most countries, is ordered by representatives of the judicial system (eg, police or court). Therefore, an autopsy report, including diagnostic results and accompanying information, is submitted to the judicial instead of the health care system. As a consequence, at least the following potential issues arise: (1) genetic information of deceased individuals resides in files of judicial institutions; (2) there is no institutional body within the judicial infrastructure who would naturally meet the medical/genetic consultation needs of the relatives, even if the relatives were to seek advice; ${ }^{6,7}$ and (3) the genetic information in the reports can be elicited to public during court proceedings. Some type of coding of the variants/ mutations in the report possibly could address privacy protection in the report (although the type of test itself may reduce the ability to protect privacy), but the proper practices of information flow from the judicial system to relatives is more cumbersome.

Second, postmortem sampling typically is a onetime opportunity. The possibility of use of histology or toxicology samples exists for some cases, but such suboptimal samples tend to be limited for large scale genomic analyses for some future question(s) that may arise. ${ }^{8}$
Possible exhumations for resampling have occurred for human identification or in the investigation of serial poisonings, ${ }^{9}$ but generally have not been entertained for other scenarios. Another situation to consider is that genetic testing may be requested only after the autopsy has been performed. ${ }^{10}$ Some standardization of collection and storage of biological specimens (eg, blood) for such purposes may facilitate effective postmortem genetic diagnostics and reports. Indeed, such protocols and suitable materials already are available and have been tested for long-term storage. ${ }^{10,11}$

Finally, medicolegal experts (forensic pathologists or medical examiners) often do not have the means, especially a timely action, to inform relatives. Many of these professionals are not trained on how to interpret the results and details in genetic reports, and genetic consultations based on these reports are not a standard part of their job description. The use of a multidisciplinary team including at least, but not limited to, a clinical geneticist and a forensic pathologist/ medical examiner is necessary to deliver proper information of a postmortem genetic test report to the relatives. ${ }^{12}$

Overall, there is a great potential value in postmortem genetic testing, which could be used effectively for the benefit of individuals, families and society. To enjoy the benefits of such testing an internationally agreed set of recommendations are needed on how to make proper use of the samples and report the results in a standardized way, a consultation team to communicate with the relative(s) of the deceased, and all practices be performed in a sensitive and private manner. Such recommendations could be added to the recommendations of the (WG) of the Genetic Services Quality Committee of the ESHG that would go far in establishing a sound and healthy foundation for postmortem medicolegal tests and the manner to report the results to all appropriate parties.

\section{CONFLICT OF INTEREST}

The authors declare no conflict of interest.

Antti Sajantila ${ }^{* 1,2}$ and Bruce Budowle 2,3
${ }^{1}$ Department of Forensic Medicine, Hjelt Institute, University of Helsinki,
Helsinki, Finland;
${ }^{2}$ Department of Molecular and Medical Genetics, Institute of Applied
Genetics, University of North Texas Health Science Center,
Fort Worth, TX, USA;
${ }^{3}$ Center of Excellence in Genomic Medicine Research (CEGMR), King
Abdulaziz University, Jeddah, Saudi Arabia
E-mail: antti.sajantila@helsinki.fi

1 Claustres M, Kozich V, Dequeker E et al: Recommendations for reporting results of diagnostic genetic testing (biochemical, cytogenetic and molecular genetic). Eur J Hum Genet 2014; 22: 160-170.

2 Tester DJ, Medeiros-Domingo A, Will ML, Haglund CM, Ackerman MJ: Cardiac channel molecular autopsy: Insights from 173 consecutive cases of autopsy-negative sudden unexplained death referred for postmortem genetic testing. Mayo Clinic Proc 2012; 87: 524-539.

3 Koren G, Cairns J, Chitayat D, Gaedigk A, Leeder SJ: Pharmacogenetics of morphine poisoning in a breastfed neonate of a codeine-prescribed mother. Lancet 2006; 368: 704.

4 Koski A, Ojanpera I, Sistonen J, Vuori E, Sajantila A: A fatal doxepin poisoning associated with a defective cyp2d6 genotype. Am J Forensic Med Pathol 2007; 28: 259-261.

5 Michaud K, Fellmann F, Abriel H, Beckmann JS, Mangin P, Elger BS: Molecular autopsy in sudden cardiac death and its implication for families: Discussion of the practical, legal and ethical aspects of the multidisciplinary collaboration. Swiss Med Wkly 2009; 139: 712-718.

6 Elger BS, Michaud K, Fellmann F, Mangin P: Sudden death: ethical and legal problems of post-mortem forensic genetic testing for hereditary cardiac diseases. Clin Genet 2010; 77: 287-292. 
7 van der Werf C, Onderwater AT, van Langen IM, Smets EM: Experiences, considerations and emotions relating to cardiogenetic evaluation in relatives of young sudden cardiac death victims. Eur J Hum Genet 2014; 22: 192-196. of cause and manner of death via a comprehensive cardiac autopsy including whole exome next-generation sequencing. Arch Pathol Lab Med 2013; 138: 1083-1089.

9 Vuori E, Pelander A, Rasanen I, Juote M, Ojanpera I: A rare case of serial killing by poisoning. Drug Test Anal 2013; 5: 725-729.
8 Loporcaro CG, Tester DJ, Maleszewski JJ, Kruisselbrink T, Ackerman MJ: Confirmation

10 Lee SB, Clabaugh KC, Silva B et al: Assessing a novel room temperature DNA storage medium for forensic biological samples. Forensic Sci Int Genet 2012; 6: 31-40.

11 Fowler KE, Reitter CP, Walling GA, Griffin DK: Novel approach for deriving genome wide snp analysis data from archived blood spots. BMC Res Notes 2012; 5: 503.

12 Kauferstein S, Kiehne N, Jenewein T et al: Genetic analysis of sudden unexplained death: a multidisciplinary approach. Forensic Sci Int 2013; 229: 122-127.

\section{Reply to Sajantila and Budowle}

European Journal of Human Genetics (2016) 24, 330; doi:10.1038/ejhg.2014.290; published online 14 January 2015

We thank Drs Sajantila and Budowle ${ }^{1}$ for raising this interesting and important topic. They correctly point out that our Recommendations for reporting results of diagnostic genetic testing ${ }^{2}$ do not cover the special circumstances surrounding the reporting of post-mortem genetic testing. We did not consider this issue while preparing our recommendations, focusing instead on reporting of routine genetic testing (biochemical, cytogenetic and molecular genetic). Although some of our recommendations will apply to all reports of genetic testing, there may be important exceptions when post-mortem results are being reported.

The issues around post-mortem genetic testing and 'molecular autopsy' go far beyond the reporting of results, encompassing inter alia issues of consent, sample integrity, legal custody and retention/storage of tissues. We understand that the Professional and Public Policy Committee (PPPC) of the ESHG is currently considering these issues with a view of producing a policy statement. The Genetics Services Quality Committee fully supports this initiative and looks forward to commenting on the draft statement when available.

\section{CONFLICT OF INTEREST}

The authors declare no conflict of interest.

David E Barton ${ }^{1}$, Mireille Claustres ${ }^{2}$, Viktor Kozich ${ }^{3}$, Els Dequeker ${ }^{4}$, Brian Fowler ${ }^{5}$, Jayne Y Hehir-Kwa ${ }^{6}$, Konstantin Miller ${ }^{7}$,

Cor Oosterwijk ${ }^{8}$, Borut Peterlin ${ }^{9}$, Conny van Ravenswaaij-Arts ${ }^{10}$, Uwe Zimmermann $^{11}$, Orsetta Zuffardi ${ }^{12}$ and Rosalind J Hastings ${ }^{\star, 13}$
${ }^{1}$ National Centre for Medical Genetics, School of Medicine \& Medical Sciences, University College Dublin,

Our Lady's Children's Hospital, Dublin, Ireland;

${ }^{2}$ Molecular Genetics of Rare Disorders, University Hospital of Montpellier and INSERM U827, IURC (Institut Universitaire de Recherche Clinique), Montpellier, France;

${ }^{3}$ Institute of Inherited Metabolic Disorders, First Faculty of Medicine, Charles University in Prague, Prague, Czech Republic;

${ }^{4}$ Biomedical Quality Assurance Research Unit, Department of Human Genetics, University of Leuven, Leuven, Belgium;

${ }^{5}$ Department of Paediatrics, Universitats-Kinderspital Beider Basel

(UKBB), Basel, Switzerland;

${ }^{6}$ Department of Human Genetics, Radboud University Medical Centre, Nijmegen, The Netherlands;

${ }^{7}$ Institute of Human Genetics, Hannover Medical School, Hannover, Germany;

${ }^{8}$ VSOP-National Patient Alliance for Rare and Genetic Disorders, Soest, The Netherlands;

${ }^{9}$ Division of Obstetrics and Gynecology, Clinical Institute of Medical Genetics, University Medical Center, Ljubljana, Slovenia;

${ }^{10}$ Department of Genetics, University of Groningen, University Medical Centre Groningen, Groningen, The Netherlands;

${ }^{11}$ Division 3 Health/Forensics, Deutsche Akkreditierungsstelle GmbH (DAkkS), Frankfurt am Main, Germany;

${ }^{12}$ Department of Medical Genetics, University of Pavia, Pavia, Italy;

${ }^{13}$ CEQAS, John Radcliffe Hospital, Oxford University Hospitals NHS Trust, Oxford, UK E-mail: Ros.Hastings@ouh.nhs.uk

1 Sajantila A, Budowle B: Postmortem medicolegal genetic diagnostics also require reporting guidance. Eur J Hum Genet 2014; e-pub ahead of print 3 December 2014; doi:10.1038/ejhg.2014.247.

2 Claustres M, Kozich V, Dequeker E et al: Recommendations for reporting results of diagnostic genetic testing (biochemical, cytogenetic and molecular genetic). Eur J Hum Genet 2014; 22: 160-170. 